\section{Interferência do vestuário no desempenho, na amplitude de movimento e no conforto na ginástica laboral}

\author{
Clothing interference in performance, articular range of motion \\ and comfort in labor gymnastics
}

1 Universidade Federal de Santa Catarina. Centro de Desportos. Laboratório de Biomecânica. Florianópolis, SC. Brasil

Recebido em 18/01/10 Revisado em 25/05/10 Aprovado em 11/06/10
Resumo - Este estudo descritivo de caso objetivou analisar a interferência do vestuário no desempenho, na amplitude de movimento (ADM) e no conforto dos exercícios físicos (EF) realizados na ginástica laboral (GL) com uniforme (UNI) e vestuário para a prática de exercícios físicos (VEF). Participaram 20 trabalhadoras com 28,9 $\pm 10,2$ anos de uma empresa de Florianópolis. Foram utilizados uma câmera fotográfica, banco de Wells, questionário e entrevista. Foram realizados sete EF: flexão do quadril, flexão do ombro, flexão do tronco-ângulos do quadril e tornozelo, extensão do ombro, flexão do tronco modificado-ângulos do quadril e tornozelo, adução horizontal do ombro e teste de sentar e alcançar com UNI e VEF. Aplicaram-se o teste t pareado, Qui-quadrado e Exato de Fisher, p $\leq 0,05$. Constatou-se que, com UNI, sete trabalhadoras cuidavam para não mostrar o corpo, 17 retiravam peças do vestuário e 13 perceberam limitação do movimento. A presença de desconforto na parte superior do corpo foi menor nos EF de flexão do quadril e adução horizontal do ombro e na parte inferior nos EF de flexão, extensão e adução horizontal do ombro. Com UNI, as ADM foram menores do que com VEF nos EF: flexão do quadril ( $p=0,017)$, flexão do ombro ( $\mathrm{p}=0,0075)$, flexão do tronco (quadril e tornozelo), flexão do tronco modificado (tornozelo), teste de sentar e alcançar (valores angulares e lineares) $(\mathrm{p}<0,001)$. O desempenho da flexão do tronco (tornozelo) foi melhor $(\mathrm{p}=0,001)$ com UNI devido ao calçado. Conclui-se que o vestuário tende a interferir na ADM e no conforto, mas não no desempenho dos exercícios físicos.

Palavras-chave: Ginástica; Trabalhadores; Amplitude de movimento articular; Vestuário.

Abstract - The objective of this descriptive case study was to analyze the interference of clothing with exercise performance, joint range of motion (ROM), and comfort during a session of labor gymnastics wearing a uniform (UNI) and specific exercise clothes (SEC). Twenty female workers (28.9 \pm 10.2 years) from a company in Florianópolis were studied. A photo camera, Wells bench, a questionnaire, and interview were used for data collection. The subjects were submitted to seven types of exercise wearing UNI and SEC: hip flexion, shoulder flexion, trunk flexion-hips and ankle angles, shoulder extension, modified trunk flexion-hips and ankle angles, horizontal shoulder adduction, and a sit-and-reach test. The results were analyzed using the paired t-test, chi-square test, and Fisher's exact test $(p \leq 0.05)$. Seven women wearing UNI tended not to show the body, 17 removed some part of UNI, and 13 noted movement limitation. Discomfort was lower in the upper body part during hip flexion and horizontal shoulder adduction and in the lower part during shoulder flexion, extension and horizontal shoulder adduction. Lower ROM values were observed for subjects wearing UNI compared to those wearing SEC during hip flexion ( $p=0.017$ ), shoulder flexion ( $p=0.0075)$, trunk flexion (hips and ankle angles), modified trunk flexion (ankle angles), and the sit-and-reach test (linear and angular values) $(p<0.001)$. Trunk flexion performance (ankle angles) was better in the UNI condition ( $p=0.001)$, probably because the subjects were wearing shoes. In conclusion, clothing tends to interfere with ROM and comfort, but not with exercise performance.

Key words: Gymnastics; Workers; Joint range of motion; Clothing. 


\section{INTRODUÇÃO}

Os programas de ginástica laboral (GL) destacam-se pela fácil implantação, baixo custo e resultados aparentemente positivos sobre a saúde do trabalhador ${ }^{1}$, apesar de existirem restrições para a sua prática, como o tempo, convencionou-se que as sessões durariam 10 minutos; o espaço, pois a GL seria realizada no próprio local de trabalho; o vestuário habitual seria mantido sem nenhuma alteração e a temperatura ambiente condicionou a intensidade dos exercícios².

Para Silva et al. ${ }^{3}$, não há necessidade de roupa especial para a prática da GL. Da mesma maneira, Sjögren et al. ${ }^{4}$ afirmam que treinamentos físicos realizados por trabalhadores, na empresa, com baixa intensidade (30\% de 1RM), não causariam suor, e desta maneira, não seria necessária a mudança do vestuário para a prática dos exercícios físicos $(\mathrm{EF})$ e tomar banho após a prática. No estudo realizado por Holmström e Ahlborg ${ }^{5}$, com trabalhadores da construção civil, foi mencionado apenas que os EF foram realizados com o vestuário habitual de trabalho, mas não o especificaram, enquanto que outros estudos ${ }^{6,7}$ não fazem menção ao vestuário utilizado pelos trabalhadores durante a GL.

Com trabalhadores de uma empresa de informática, constatou-se que o vestuário não foi citado por nenhum trabalhador como causa da não-adesão ao programa, no entanto, foi mencionada a inadequação do ambiente e o constrangimento, devido à exposição pessoal no local de trabalho ${ }^{8}$. Apesar das variáveis de interferência, houve aceitação dos trabalhadores ao programa e estes deram credibilidade à eficiência de curtos períodos de atividade física durante o trabalho. Por outro lado, um estudo sobre a forma como os programas de GL eram realizados destaca que a prática de EF com roupas de trabalho em um país tropical, como o Brasil, pode causar desconfortos, principalmente, associados a não utilização de uniformes (UNI) ou a não troca de roupas para trabalhar'.

Na elaboração de um programa de GL, é necessário que os exercícios respeitem o vestuário dos trabalhadores ${ }^{8,10}$. Todavia, observou-se que alguns estudos ${ }^{5,6,7}$ não trazem orientações aos profissionais de Educação Física quanto às recomendações que estes devam repassar aos trabalhadores, diariamente, sobre o vestuário, tais como: retirar peças do vestuário e realizar os EF na amplitude que o vestuário permitir, proporcionando conforto e satisfação aos trabalhadores.

A implementação dos programas de GL tem aumentado no Brasil, mas existem poucas evidências sobre os seus possíveis benefícios, como a melhora da amplitude de movimento (ADM), haja vista os estudos que não controlam algumas variáveis (frequência no programa e a prática de atividade física fora do local de trabalho) para poder comprovar a relação entre a participação na GL e a melhora da $\mathrm{ADM}^{9}$ e a interferência do UNI na ADM.

Sendo assim, este estudo tem como objetivo analisar a interferência do vestuário no desempenho, na ADM e no conforto percebido pelas trabalhadoras durante os EF realizados na GL, além de analisar o nível de satisfação e a percepção das trabalhadoras quanto à importância do programa de ginástica laboral.

\section{PROCEDIMENTOS METODOLÓGICOS}

Este estudo descritivo do tipo estudo de caso foi aprovado pelo Comitê de Ética para Pesquisas com Seres Humanos da Universidade Federal de Santa Catarina - UFSC (Processo no 032/2008). Participaram 20 trabalhadoras de uma empresa privada de prestação de serviços de Florianópolis, SC, Brasil, com 28,9 $\pm 10,2$ anos de idade. A escolha das participantes foi não-probabilística intencional por voluntariado, tendo como critérios de inclusão: a utilização do UNI fornecido pela empresa e a participação no programa de GL a pelo menos um mês.

A coleta de dados ocorreu em uma sala da empresa a $22,8 \pm 0,4^{\circ} \mathrm{C}$. Participavam do programa de GL 40 trabalhadoras (22 vestiam UNI durante o trabalho), a GL ocorria simultaneamente com as atividades desenvolvidas em dois setores: refeitório e agendamento, ambos climatizados.

O programa de GL era desenvolvido há cinco anos e três vezes por semana nessa empresa; utilizavam-se técnicas de alongamento, fortalecimento muscular, massagem, entre outros; com música e diferentes materiais; produziam-se reportagens semanais sobre saúde; avaliações físicas anuais; orientações posturais e trekking uma vez ao ano.

Os instrumentos de medidas utilizados foram: câmera fotográfica Samsung ${ }^{\circledR}$ S85, com resolução de 8.2 mega pixels, posicionada perpendicularmente a 2,5 $\mathrm{m}$ de distância das avaliadas, fixada em um tripé a 91,5 cm e $47 \mathrm{~cm}$ de altura (posição corporal em pé e sentada, respectivamente), e para a análise das imagens (ADM), utilizou-se o Software CorelDraw $^{\circledR}$ 9.0; banco de Wells para realizar o teste de sentar e alcançar; questionário contendo informações sobre: 1) dados de identificação, 2) 
percepção das trabalhadoras quanto às características do vestuário, 3) satisfação e importância do programa de GL; e entrevista estruturada para verificar a dificuldade em realizar os EF e o conforto do vestuário (parte superior e inferior da roupa e calçado). O questionário e a entrevista estruturada obtiveram índice de validade de 93 e 92\% e clareza de 94 e $91 \%$, respectivamente.

Após as trabalhadoras concordarem em participar da pesquisa, assinaram o termo de consentimento livre e esclarecido, e no dia agendado, dirigiram-se a uma sala da empresa, uniformizadas (calça e colete de poliéster - tecido sintético - e camisa fornecida ou recomendada pela empresa) para a coleta de dados. Com UNI, as trabalhadoras usaram o calçado que estavam habituadas a trabalhar (salto do tipo anabela com $1 \mathrm{~cm}$ na parte anterior e $4 \mathrm{~cm}$ na posterior), como o uso do calçado fornecido pela empresa não era obrigatório, foram utilizados diferentes calçados. As alturas dos solados dos calçados, na parte anterior, variaram de 1 a $5,5 \mathrm{~cm}$ com UNI e com vestuário para a prática de exercícios físicos (VEF) de 0 a 2,0 cm, sendo que, na parte posterior dos calçados, a variação foi de 0 a $8,5 \mathrm{~cm}$ e 0 a $4,0 \mathrm{~cm}$, respectivamente. Portanto, as diferenças entre as alturas anterior e posterior dos calçados tanto com UNI como com VEF variaram de 0 a $4,0 \mathrm{~cm}$. Foi recomendado às trabalhadoras que retiravam os calçados rotineiramente durante a GL, que o fizessem na coleta, e que trouxessem um VEF (uma calça com tecido flexível e justa ao corpo, camiseta sem manga, meia e tênis).

Primeiramente, as trabalhadoras responderam o questionário, e logo após, receberam as explicações sobre os procedimentos da coleta de dados: 1) marcação dos pontos anatômicos - devido aos resultados do estudo piloto do presente estudo, adotou-se a marcação dos pontos anatômicos modificados com UNI, na qual os pontos anatômicos (acrômio, epicôndilo lateral do úmero, trocânter maior, epicôndilo lateral do fêmur, maléolo la- teral e cabeça do $2^{\circ}$ metatarso) eram marcados no hemicorpo direito sobre o UNI, por meio de palpação na postura de ADM máxima; com VEF os pontos anatômicos foram marcados e não eram modificados após os EF; 2) captura das imagens - a trabalhadora deveria permanecer na posição estática até a captura de três fotografias consecutivas em cada EF na ADM máxima.

As trabalhadoras realizaram sete EF (Figura 1), intercalando membros inferiores e superiores, primeiramente, com UNI e depois com VEF. Após cada $\mathrm{EF}$, as trabalhadoras respondiam duas perguntas sobre dificuldade em realizar o EF e o conforto do vestuário.

No teste de sentar e alcançar, as trabalhadoras realizaram três tentativas, com intervalo de 20 segundos e selecionou-se a fotografia correspondente ao maior valor no teste para realizar as análises angulares. Foram utilizados a estatística descritiva, teste t pareado, Qui-quadrado e Exato de Fisher, $\mathrm{p} \leq 0,05$.

\section{RESULTADOS}

A maioria das trabalhadoras percebeu as interferências do UNI, pois 13 sentiram limitação do movimento ao realizarem a GL, algumas realizavam os EF com cautela para não mostrar alguma parte do corpo (7) e retiravam peças do UNI (17) para realizarem a GL. Apesar da maioria (19) ter percebido, na posição fundamental, o tamanho do UNI como adequado, as regiões das coxas/axilas e ombros foram as partes do corpo que mais apertavam as trabalhadoras durante a GL.

Na Tabela 1, estão dispostos os valores das $\mathrm{ADM}$ nos sete $\mathrm{EF}$ analisados, nos quais foram avaliadas as articulações do ombro, quadril e tornozelo.

Os resultados mostram que houve diferença significativa entre as ADM nos EF: flexão do quadril ( $p=0,017)$, flexão do ombro $(p=0,0075)$, flexão do tronco (quadril e tornozelo), flexão do tronco

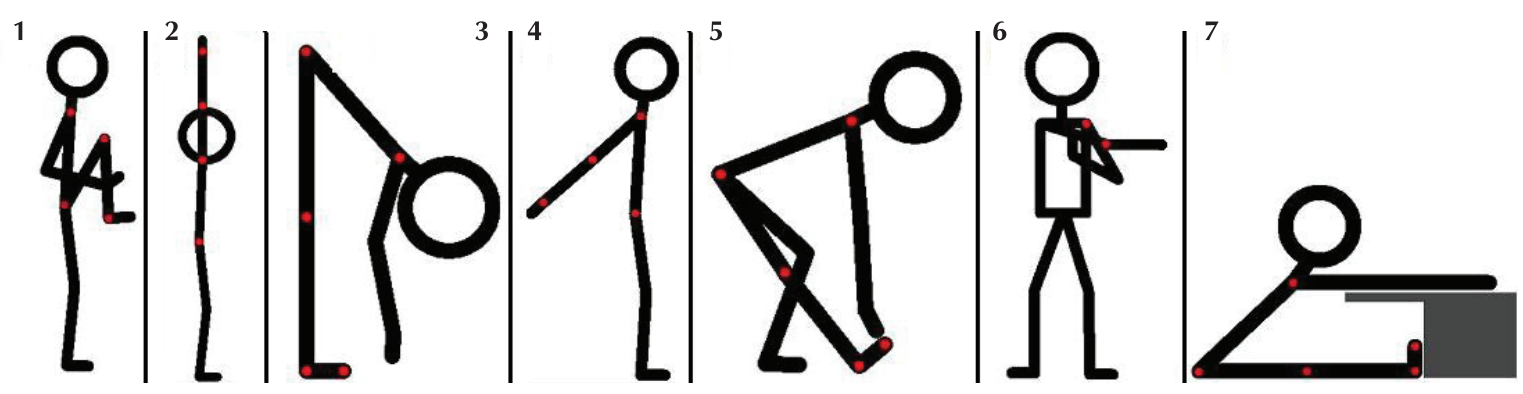

Figura 1. Sequência dos EF: 1) Flexão do quadril; 2) Flexão dos ombros; 3) Flexão do tronco (ângulos do quadril e tornozelo); 4) Extensão dos ombros; 5) Flexão de tronco modificado (ângulos do quadril e tornozelo); 6) Adução horizontal do ombro (valores lineares); 7) Teste de sentar e alcançar (valores angulares e lineares). *Os círculos representam os pontos anatômicos. 
Tabela 1. Valores angulares, média \pm desvio-padrão, mínimo, máximo, diferença dos valores angulares entre UNI e VEF e comparação das ADM nos dois vestuários em cada EF.

\begin{tabular}{|c|c|c|c|c|}
\hline Exercícios físicos & $\bar{X} \pm \mathrm{S}\left({ }^{\circ}\right)$ & Mín. - Máx. $\left(^{\circ}\right)$ & $\mathrm{T}$ & $\mathrm{p}$ \\
\hline UNI & $106,9 \pm 6,0$ & $93,0-118,3$ & \multirow{3}{*}{2,284} & \multirow{3}{*}{$0,017^{*}$} \\
\hline VEF & $110,0 \pm 7,0$ & $99,1-122,8$ & & \\
\hline$\neq\left({ }^{\circ}\right)^{\dagger}$ & $3,1 \pm 6,1$ & $-8,9-18,3$ & & \\
\hline \multicolumn{5}{|l|}{ Flexão do ombro } \\
\hline VEF & $148,4 \pm 6,0$ & $138,7-162,4$ & \multirow{2}{*}{$-2,688$} & \multirow{2}{*}{$0,0075^{*}$} \\
\hline$\left.\neq^{\circ}\right)^{+}$ & $2,7 \pm 4,4$ & $-7,0-11,3$ & & \\
\hline \multicolumn{5}{|c|}{ Flexão do tronco - quadril } \\
\hline UNI & $100,7 \pm 14,9$ & $68,4-119,9$ & $-4,358$ & $<0,001^{* *}$ \\
\hline UNI & $24,1 \pm 3,9$ & $18,7-33,2$ & \multirow{3}{*}{3,886} & \multirow{3}{*}{$<0,001^{* *}$} \\
\hline VEF & $19,4 \pm 3,8$ & $12,7-27,0$ & & \\
\hline$\neq\left(^{\circ}\right)^{+}$ & $4,7 \pm 5,4$ & $-5,4-19,1$ & & \\
\hline \multicolumn{5}{|c|}{ Extensão do ombro } \\
\hline UNI & $41,9 \pm 11,1$ & $22,5-65,7$ & \multirow{3}{*}{$-1,286$} & \multirow{3}{*}{0,107} \\
\hline VEF & $43,3 \pm 11,5$ & $26,2-60,3$ & & \\
\hline$\neq\left({ }^{\circ}\right)^{+}$ & $1,4 \pm 4,8$ & $-6,4-10,2$ & & \\
\hline \multicolumn{5}{|c|}{ Flexão do tronco modificado - quadril } \\
\hline VEF & $-1,4 \pm 5,4$ & $-13,3-8,3$ & \multirow{3}{*}{3,827} & \multirow{2}{*}{$<0,001^{* *}$} \\
\hline$\neq\left({ }^{\circ}\right)^{+}$ & $4,8 \pm 5,6$ & $-3,6-18,0$ & & \\
\hline \multicolumn{4}{|c|}{ Adução horizontal do ombro } & \\
\hline UNI & $17,3 \pm 2,5$ & $12,5-21,7$ & \multirow{3}{*}{1,555} & \multirow{3}{*}{0,068} \\
\hline VEF & $16,7 \pm 2,4$ & $11,6-22,9$ & & \\
\hline$\neq(\mathrm{cm})^{+}$ & $0,7 \pm 1,9$ & $-3,2-3,2$ & & \\
\hline \multicolumn{3}{|c|}{ Teste de sentar e alcançar - quadril } & \multirow{4}{*}{3,928} & \\
\hline UNI & $112,2 \pm 14,9$ & $88,4-134,7$ & & \\
\hline VEF & $116,5 \pm 14,4$ & $82,1-138,8$ & & $<0,00 T^{* *}$ \\
\hline$\neq\left({ }^{\circ}\right)^{+}$ & $4,3 \pm 4,9$ & $-6,8-13,3$ & & \\
\hline Teste de sentar e a & & & & \\
\hline UNI & $21,2 \pm 11,7$ & $0-35,5$ & 5197 & $<00 \Omega 1 * *$ \\
\hline VEF & $24,7 \pm 11,4$ & $0-40,0$ & $-5,191$ & $<0,001$ \\
\hline$\neq(\mathrm{cm})^{+}$ & $3,5 \pm 3,0$ & $-1,0-8,0$ & & \\
\hline
\end{tabular}

${ }^{*} \mathrm{p}<0,05 ; * \mathrm{p}<0,001 ;{ }^{+} \mathrm{O}$ valor negativo significa que a medida angular ou linear com o VEF obteve desempenho inferior em relação ao UNI; UNI - uniforme; VEF - Vestuário para a prática de exercícios físicos; Teste t pareado.

modificado (tornozelo), teste de sentar e alcançar (quadril e valores lineares $-\mathrm{p}<0,001$ ); sendo que com UNI as ADM foram significativamente menores do que com $\mathrm{VEF}$.

A maior parte $(44,4 \%)$ das diferenças das médias dos valores angulares variou de $0^{\circ}$ a $5^{\circ}$ e dos valores lineares de 0 a 5,0 cm. Após a análise das
160 ADM (angulares e lineares), verificou-se que $78 \%$ apresentaram valores inferiores com UNI que com VEF, mostrando a interferência do UNI na ADM nos EF realizados.

Os desempenhos físicos das trabalhadoras, utilizando os dois vestuários ao realizarem os cinco EF, estão apresentados na Tabela 2. 
Tabela 2. Comparação do desempenho físico (ADM) das trabaIhadoras utilizando os dois vestuários ao realizarem cinco EF.

\begin{tabular}{|c|c|c|c|}
\hline ADM & UNI & VEF & $P$ \\
\hline \multicolumn{4}{|c|}{ Extensão do ombro } \\
\hline$<45^{\circ}$ & 12 & 11 & \multirow{2}{*}{0,749} \\
\hline$\geq 45^{\mathrm{oK}}$ & 8 & 9 & \\
\hline \multicolumn{4}{|l|}{ Flexão do ombro } \\
\hline$<180^{\circ}$ & 20 & 20 & \multirow{2}{*}{-} \\
\hline$\geq 180^{\circ} \mathrm{K}$ & $0^{\mathrm{a}}$ & $0^{\mathrm{a}}$ & \\
\hline \multicolumn{4}{|c|}{ Flexão do quadril (ângulo do quadril) } \\
\hline$<110^{\circ}$ & 13 & 10 & \multirow{2}{*}{0,337} \\
\hline$\geq 110^{\circ R}$ & 7 & 10 & \\
\hline \multicolumn{4}{|c|}{ Flexão dorsal do tornozelo - Flexão do tronco } \\
\hline$<20^{\circ}$ & 3 & 13 & \multirow{2}{*}{$0,001^{*}$} \\
\hline$\geq 20^{\circ \mathrm{K}}$ & 17 & 7 & \\
\hline \multicolumn{4}{|c|}{ Flexão dorsal do tornozelo - Flexão do tronco modificado } \\
\hline$<20^{\circ}$ & 20 & 20 & \\
\hline$\geq 20^{\circ \mathrm{K}}$ & $0^{\mathrm{a}}$ & $0^{\mathrm{a}}$ & \\
\hline \multicolumn{4}{|c|}{ Teste de sentar e Alcançar } \\
\hline Abaixo da média & 17 & 12 & \multirow{2}{*}{0,077} \\
\hline Acima da média & 3 & 8 & \\
\hline
\end{tabular}

Teste Qui-quadrado; Recomendações de ADM classificadas

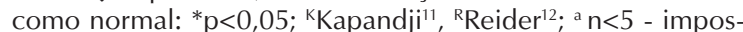
sibilitou a realização do teste estatístico; UNI - uniforme; VEF - Vestuário para a prática de exercícios físicos.

Não existiu diferença significativa entre o uso do vestuário e o desempenho da ADM para os EF: extensão do ombro, flexão do quadril e teste de sentar e alcançar, pois os dados comportaram-se de maneira semelhante nas duas situações (Tabela 2), havendo diferença significativa ( $\mathrm{p}=0,001)$ no desempenho da flexão dorsal do tornozelo no EF de flexão do tronco.

As trabalhadoras perceberam dificuldade significativamente maior apenas ao realizarem a flexão do quadril com UNI, quando comparado com o VEF ( $p=0,003$ ), não havendo diferença significativa nos demais EF.

A parte superior do vestuário (UNI) interferiu na limitação do movimento e conforto tanto nos EF para os membros inferiores como para os membros superiores (flexão, extensão e adução horizontal do ombro, flexão do tronco, flexão do tronco modificado e teste de sentar e alcançar) ( $<<0,05)$. Já a parte inferior do vestuário causava desconforto nos EF que envolviam os membros inferiores (flexão do quadril, flexão do tronco, flexão do tronco modificado e teste de sentar e alcançar) $(\mathrm{p}<0,05)$, entretanto, o desconforto do calçado foi percebido apenas no EF de flexão de tronco modificado $(\mathrm{p}=0,047)$.

Constatou-se que não houve associação (teste Exato de Fisher) do desempenho com o conforto ao realizar os EF: extensão de ombro $(\mathrm{p}=0,351)$, flexão do quadril $(p=0,524)$ e flexão dorsal do tornozelo no EF de flexão do tronco $(\mathrm{p}=0,116)$.

Todas as trabalhadoras estavam satisfeitas com o programa de GL e classificaram-no como importante, citando que o programa é uma maneira de melhorar a saúde, a postura, promover a prática de EF, combater o sedentarismo, evitar doenças ocupacionais e aumentar a disposição para o trabalho.

\section{DISCUSSÃO}

As trabalhadoras perceberam interferência negativa do UNI durante a GL, pois 13/20 sentiam limitação do movimento e todas justificaram que o tecido do uniforme não possuía elasticidade. A limitação do movimento poderia estar relacionada com os EF prescritos e/ou com o conforto percebido em relação ao vestuário, pois no estudo de Martins $^{13}$, ao verificar a usabilidade e o conforto de dois UNI, realizando diferentes tarefas, inclusive alongamentos, foi constatado que os tecidos destinados à confecção de UNI devem ser menos sintéticos, proporcionando maior mobilidade ao usuário e com acabamentos de materiais maleáveis.

A percepção da limitação do movimento pelas trabalhadoras contrapõe um dos objetivos do EF mais aplicado na $\mathrm{GL}^{1,3,5-7}$, o alongamento, que tem a função de aumentar a ADM ${ }^{14,15}$. Apesar disso, apenas uma trabalhadora deixou de participar da GL devido à roupa que estava vestindo, o mesmo foi confirmado em outro estudo ${ }^{8}$, no qual o vestuário não foi citado como causador da não-adesão ao programa.

A prescrição dos EF na GL deve respeitar o vestuário utilizado pelos trabalhadores ${ }^{10}$, mas faltam informações para orientarem os profissionais de Educação Física a promoverem conforto e aumento da ADM na GL, como a retirada de peças do vestuário. Maciel et al. ${ }^{9}$ mencionam que a prática de EF com roupa de trabalho é muito desconfortável, principalmente, em um país tropical e também, para aqueles trabalhadores que não utilizam uniformes de trabalho ou não efetuam troca de roupas para trabalhar. No presente estudo, a GL era praticada em ambientes climatizados, com o objetivo de prevenir a sudorese e o desconforto dos trabalhadores.

Verificou-se que das dez ADM analisadas, sete foram significativamente menores com UNI do que com VEF e a maior parte das diferenças entre os valores angulares variou de 0 a $5^{\circ}$ e os lineares de 0 a $5 \mathrm{~cm}$. Observou-se uma carência de estudos quan- 
titativos que abordem a limitação do movimento devido ao vestuário na prática da GL, sendo que se encontrou apenas estudos qualitativos ${ }^{8,9}$.

No presente estudo, os valores do teste de sentar e alcançar foram de $21,2 \pm 11,7 \mathrm{~cm}$ com UNI e $24,7 \pm 11,4 \mathrm{~cm}$ com VEF, sendo que houve diferença significativa $(p<0,001)$ entre os valores angulares do quadril e lineares do teste com os dois vestuários (Tabela 1). Santos e Moro $^{16}$ verificaram valores semelhantes $(24,5 \pm 7,25 \mathrm{~cm}$ no pré-teste e $25,53 \pm$ $7,99 \mathrm{~cm}$ no pós-teste) nesse teste com 16 trabalhadores submetidos a um programa de GL, todavia, os trabalhadores estavam de sunga ou maiô. Em outro estudo $^{7}$, com 26 servidores públicos, os valores do teste foram de 24,21 $\pm 9,49 \mathrm{~cm}$ no pré-teste e 26,24 $\pm 9,36 \mathrm{~cm}$ no pós-teste, não sendo mencionado o vestuário utilizado pelos trabalhadores.

Apesar de haver diferença significativa entre os valores do teste de sentar e alcançar, não houve diferença entre as duas classificações (agrupadas) no desempenho das trabalhadoras com os dois vestuários. Por esse motivo, torna-se necessária a avaliação de um número maior de indivíduos para possibilitar a análise estatística nas cinco classificações do teste de sentar e alcançar, e assim, verificar se o UNI não interfere no desempenho do avaliado.

Os diferentes tipos de calçados utilizados podem ter influenciado no desempenho da flexão dorsal do tornozelo no EF de flexão do tronco. Segundo Sacco et al. ${ }^{17}$, o salto alto exige alterações posturais a fim de manter o equilíbrio postural ocasionado pelo deslocamento do centro de gravidade para frente, colocando o corpo sob condições não-fisiológicas, alterando a função das articulações, gerando forças e torques acima dos normais. Para Santos et al. ${ }^{18}$, a altura do salto do calçado é diretamente proporcional à intensidade das alterações biomecânicas.

No estudo atual, ocorreram alterações angulares no tornozelo, nos EF de flexão do tronco e flexão do tronco modificado, o que é explicado por Ebbeling et al. ${ }^{19}$, pois o uso de salto gera desequilíbrio muscular, o que afeta o funcionamento de toda a cadeia cinética do membro inferior, principalmente, dos tornozelos, pelo aumento da flexão plantar. As alturas dos saltos com UNI e VEF variaram de 0 a $4 \mathrm{~cm}$, não ultrapassando a altura $(5 \mathrm{~cm})$ do calçado feminino recomendada para manter o conforto e reduzir os riscos de lesões ${ }^{19}$.

As trabalhadoras perceberam dificuldade significativamente maior ao realizarem a flexão do quadril com UNI do que com VEF, pois a calça apertava (6) e havia dificuldade em manterem-se equilibradas (3), já que este $\mathrm{EF}$ é realizado sobre o apoio de apenas um pé no solo.

Uma trabalhadora ao realizar os EF descalça (UNI) percebeu desconforto no calcanhar apenas na flexão do tronco modificado, pois parte da massa corporal estava apoiada sobre o calcanhar do membro inferior anteriorizado.

No estudo atual, todas as trabalhadoras mostraram-se satisfeitas com o programa de GL. Resultado semelhante ocorreu no estudo de Trujillo e Zeng ${ }^{6}$, que apesar dos alongamentos serem demonstrados por um software (Stop and Strech) na tela do microcomputador (micro-pausas), a cada 60 minutos, 94,7\% dos trabalhadores estavam satisfeitos com o uso desse recurso visual.

O programa de GL foi percebido por todas as trabalhadoras como importante para a saúde, da mesma maneira, alguns estudos confirmam os seus benefícios ${ }^{7,20}$, no entanto, outros questionam a sua efetividade ${ }^{9,21,22}$, sugerindo a realização de estudos com amostras representativas e delineamentos mais robustos para constatação dos referidos benefícios, como o aumento da ADM.

Holmström e Ahlborg ${ }^{5}$ constataram que exercícios de aquecimento de curta duração pela manhã, podem ser eficientes para aumentar ou manter a ADM e aumentar a resistência dos músculos das costas dos trabalhadores que realizem funções manuais e adotem posturas extremas durante o trabalho. Em contrapartida, os mesmos autores citam as limitações do estudo: design não-randomizado, dificuldade dos trabalhadores participarem do estudo e examinadores não cegos em relação aos grupos experimentais. Já os resultados do estudo de Paiva Neto et al..$^{23}$, possibilitam afirmar que a GL pode contribuir para a manutenção da ADM, que está diretamente relacionado com a duração e a frequência das sessões, pois os trabalhadores participavam do programa, há 9 anos, com cinco sessões semanais. Ao realizarem uma revisão sistemática, Coury, Moreira e Dias ${ }^{22}$ concluíram que os estudos sobre GL com alta qualidade metodológica e resultados efetivos foram aqueles em que o tempo de aplicação do protocolo era igual ou superior a dez semanas.

A não-padronização do vestuário usado em empresas pode ter efeitos negativos no programa de GL, pois segundo Maciel et al. ${ }^{9}$, só o fato dos trabalhadores exercitarem-se na frente de seus colegas e chefes já está associado a desconfortos. Todavia, as autoras não citam as condições que poderão proporcionar conforto aos trabalhadores, como a postura profissional e a maneira como as sessões de GL são conduzidas (seleção dos exercícios e orientações com relação às roupas utilizadas pelas 
trabalhadoras - saias, vestidos e blusas decotadas), além de generalizarem que o relacionamento entre os trabalhadores, na sua maioria, é ruim. Por outro lado, no presente estudo, as trabalhadoras mencionaram estarem satisfeitas com o programa, pois sentiam-se à vontade com a profissional de GL e com as colegas de trabalho durante as sessões.

Para Devide ${ }^{24}$, no momento da implantação da GL, os diferentes fatores motivacionais que levam à prática de EF devem ser estudados, pois o que pode satisfazer uma pessoa pode desconfortar outras. De acordo com Soares, Assunção e Lima ${ }^{25}$, uma das explicações para a baixa adesão à GL pode ser a falta de orientação e acompanhamento dos instrutores ao programa e a emulação social entre o instrutor e trabalhadores.

Com base nestas afirmações, talvez a prática de GL por trabalhadores que utilizem uniformes seja mais apropriada, pois caso contrário, cada trabalhador adotará um vestuário diferente, o que poderia limitar ainda mais a prescrição dos EF na GL.

\section{CONCLUSÃO}

O uniforme interferiu negativamente na amplitude de movimento e na percepção de conforto das trabalhadoras ao realizarem sete exercícios físicos, durante a ginástica laboral, mas não influenciou no desempenho dos exercícios físicos e mesmo as trabalhadoras percebendo a limitação do movimento devido ao uniforme, estavam satisfeitas com o programa, e não consideraram o vestuário um empecilho para a prática.

Os resultados deste estudo podem contribuir para orientar: os profissionais de Educação Física quanto à prescrição dos exercícios físicos na ginástica laboral em relação ao vestuário utilizado pelos trabalhadores; as empresas que desenvolvem ou desejam implantar programa de ginástica laboral, em relação à aquisição de uniformes confeccionados com tecidos que proporcionem mobilidade articular e sejam confortáveis ou a adoção de normas quanto ao vestuário utilizado pelos trabalhadores na empresa; e por último, as empresas que confeccionam uniformes, no sentido de adaptarem-se às novas culturas empresariais e rotinas laborais, visando proporcionar maior conforto aos trabalhadores durante a ginástica laboral.

\section{REFERÊNCIAS BIBLIOGRÁFICAS}

1. Resende MCF, Tedeschi CM, Bethônico FP, Martins TTM. Efeitos da ginástica laboral em funcionários de teleatendimento. Acta Fisiatr 2007;14(1):25-31.
2. Faria Júnior, AG. Educação Física no mundo do trabalho: Ginástica de Pausa, em busca de uma metodologia. In: Quintas G. Esporte e Lazer na Empresa. Brasília: MEC; 1990.

3. Silva J, Taranto IC, Piasecki F. Ginástica Laboral: alongamento x flexionamento. SaBios-Rev. Saúde e Biol 2006;1(2):6-12.

4. Sjögren T, Nissinen KJ, Järvenpãã SK, Ojanen MT, Vanharanta H, Mälkiä EA. Effects of a physical exercise intervention on subjective physical well-being, psychosocial functioning and general well-being among office workers: A cluster randomized-controlled cross-over design. Scand J Med Sci Sports 2006;16(6):381-90.

5. Holmström E, Alhborg B. Morning warming-up exercise - effects on musculoskeletal fitness in construction workers. Appl Erg 2005;36:513-519.

6. Trujillo L, Zeng X. Data entry workers perceptions and satisfaction response to the "Stop and Stretch" software program. Work 2006;27:111-21.

7. Martins CO, Duarte MFS. Efeitos da ginástica laboral em servidores da Reitoria da UFSC. Rev Bras Ciênc Mov 2000;8(4):7-14.

8. Moser AD, Alves JPV, Albuquerque 1CA. Motivando para a saúde. Rev Proteção 2003;143:66-69.

9. Maciel RH, Albuquerque AMFC, Melzer AC, Leônidas SR. Quem se Beneficia dos Programas de Ginástica Laboral? Cad Psicol Soc Trab 2005;8:71-86.

10. Nunes R, Tirloni AS. UDESC na Empresa - Programa de Atividade Física (Artigo). II Congresso Brasileiro de Extensão Universitária. Belo Horizonte: 2004; Disponível em: <http://www.ufmg.br/congrext/Saude/ Saude191.pdf> [2006 jun 29].

11. Kapandji, IA. Fisiologia articular: esquemas comentados de mecânica humana. 5.ed. São Paulo: Médica Panamericana, 3 v.; 2000.

12. Reider, B. O exame Físico em ortopedia. Rio de Janeiro: Guanabara Koogan; 2001.

13. Martins SB. O conforto no vestuário: uma interpretação da ergonomia. Metodologia para avaliação de usabilidade e conforto no vestuário. [Tese de Doutorado - Programa de Pós-Graduação em Engenharia de Produção] Florianópolis (SC): Universidade Federal de Santa Catarina; 2005.

14. Rosário JLR, Marques AP, Maluf AS. Aspectos clínicos do alongamento: uma revisão de literatura. Rev Bras Fisioter 2004;8(1):83-8.

15. Knudson D. Stretching: from science to practice. JOPERD 1998;69(3):38-42.

16. Santos JB, Moro ARP. Programa de Exercício Físico na Empresa (PEFE): um estudo com trabalhadores de informática. Rev Bras Fisiol Exerc 2006;5:42-9.

17. Sacco ICN, Melo MCS, Rojas GB, Naki IK, Burgi K, Silveira LTY, et al. Análise biomecânica e cinesiológica de posturas mediante fotografia digital: estudo de casos. Rev Bras Ciênc Mov 2003;11(2):24-33.

18. Santos CL, Noronha DO, Gomes CA, Fernandes PR, Fernandes Filho J. Repercussões biomecânicas do uso de salto alto na cinemática da marcha: um estudo retrospectivo de 1990 a 2007. Rev Ed Fís 2008;143:47-53.

19. Ebbeling CJ, Hamill J, Crussemeyer JA. Lower extremity mechanics and energy cust of walking in high-heeled shoes. J Orthop Sposts Phys Ther 1994;19:190-6. 
20. Proper KI, Heymans MW, Paw MJMCA, Sluijs EMFV, Poppel MNMV, Mechelen WV. Promoting physical activity with people in different places - A dutch perspective. J Sci Med Sport 2006;9:371-7.

21. Dishman RK, Oldenburg B; O'neal H, Shephard RJ. Worksite physical activity interventions. Am J Prev Med 1998;15(4):344-61.

22. Coury, HJCG, Moreira, RFC, Dias, NB. Efetividade do exercício físico em ambiente ocupacional para controle da dor cervical, lombar e do ombro: uma revisão sistemática. Rev Bras Fisioter 2009;13(6):461-79.

23. Paiva Neto A, Barbosa CGR, Abdala DW, Silva Júnior AJ, Mendes MS. Perfil da flexibilidade em trabalhadores participantes de um programa de ginástica laboral de uma empresa metalúrgica de Guaxupé - MG. Fit Perf J 2009;8(4):279-85.

24. Devide FP. Atividade física na empresa: para onde vamos e o que queremos? Motriz 1998;4(2):109-15.
25. Soares RG, Assunção AÁ, Lima FPA. A baixa adesão ao programa de ginástica laboral: buscando elementos do trabalho para entender o problema. Rev Bras Saúde Ocup 2006;31(114):149-60.

\section{Endereço para correspondência} Adriana Seára Tirloni

Rua: Baraúna, 159 - Parque São Jorge Florianópolis - SC - CEP: 88034-450

E-mail: adri@tirloni.com.br 